\title{
The Types Division and Severity Threshold of Traffic Conflict on Freeway Interchange
}

\author{
Shen Li, Qiaojun Xiang, Xin Gu, Junchen Dai, Chao Tang and Shipeng Zhao \\ School of Transportation, Southeast University, Si Pai Lou \#2, Nanjing 210096, China
}

\begin{abstract}
This paper selects time to collision (TTC) as the traffic conflict index. Shooting high-definition video from high altitude above freeway interchange by unmanned aircraft, the TTC was calculated by processing software. The mechanism of traffic conflict on Freeway Interchange area was researched by combining subjective and objective method, and then defined severity. Firstly, conflict observers were trained. Twelve observers were trained repeatedly on the judgment of traffic conflict type and severity cognitive until a consensus was reached. Secondly, the subjective judgment on traffic conflict types and severity was made. The video clips containing traffic conflict were selected, and then all observers together determined the type of each conflict and severity. Thirdly, TTC samples were analyzed. The obviously unsuitable TTC samples were excluded, and the rest would be tested whether meet the sample size requirement of each type of traffic conflict. Finally, the TTC threshold value was defined. According to the cumulative frequency distribution of each type TTC, $85 \%$ value was selected as severity of traffic conflict threshold.
\end{abstract}

\section{Introduction}

Freeway interchange, including diverge, merge, and weaving area, is the traffic facilities providing continuous traffic flow. However, interference is still existed in traffic flow, which often leads to sideswipe and rear-end accidents. The traditional traffic accident statistics record has the defect of lacking in standardization and systematisms, and the small sample size. Traffic Conflict could be observed with high efficiency, and could be given quantitative analyzed. Itis a Traffic safety Evaluation Index of non-accident [1]. Since the 1980s, the technology has been extensive researched and applied in Intersection Traffic Safety evaluation in China [2], [3], W. Cheng, improved the traffic safety of urban road intersection with traffic conflict [4], [5], Q. Xiang, improved the highway intersection [6], [7], F. Pan, analyzed the unsignalized intersection[8], W. Guo renovated the traffic conflict discrimination model[9]. However, it lacks research on application in the Interchange. And before applying traffic conflict, the criteria traffic conflict must be determined primarily, including conflict type division and define the severity rating.

In order to ensure the traffic flow video quality, highaltitude unmanned aircraft hanging shot was used to overcome two problems. One was that traffic conflict points occurred were not fixed in interchange area. The other was that the digital video could not be placed because there was no high-rise building around interchange.
Tracker is a free video analysis and modeling tool built on the Open Source Physics (OSP) Java framework. Features include object tracking with position, velocity and acceleration overlays and graphs, special effect filters, multiple reference frames, calibration points, line profiles for analysis of spectra and interference patterns, and dynamic particle models. It is designed to be used in introductory college physics labs and lectures.

In this paper, determining the type division and definition severity of traffic conflict of freeway interchange was dealt with the method of combining objective and subjective analysis. TTC was selected as traffic conflict's evaluation index. Firstly, the severity and type of traffic conflict was subjectively judged by 12 trained observers. And conflict threshold was defined objectively by statistical analysis, then the safety evaluation method of interchange was formed which based on traffic conflict technology.

\section{Lecture review}

\subsection{Traffic safety evaluation method}

There were two evaluation traffic safety methods of freeway, the speed consistency and comprehensive evaluation.

\subsubsection{The speed consistency}


T. Guo, safety performance of 37 exit ramps are appraised using three different measures namely $\Delta \mathrm{V} 85$, $85(\Delta \mathrm{V})$, and $85(\Delta \mathrm{V}) \mathrm{R}$. The result show that $85(\Delta \mathrm{V})$ is 1.42 to 2.02 times of $\Delta \mathrm{V} 85$, and that the evaluation result of $85(\Delta \mathrm{V}) \mathrm{R}$ was between the other two [10]. Y. Zhang, built specific route design process based on the operating speed consistency, according to the service level of freeway safety, constructed alignment design consistency evaluation and evaluation criteria, and finally the use of research results for a proposed highway route design consistency analysis [11]. W. Wu, according to the interchange ramp speed variation, the ramp was divided into three sections: the deceleration section, uniform section, and the acceleration section. Analyze the relationship between speed change and influencing factors between the various segments, determined interchange ramp speed prediction correlation coefficient factors to construct interchange ramp operating speed prediction model for each segment. And prediction models for the relevant test to verify the applicability of the model [12].

\subsubsection{Comprehensive evaluation}

S. Fan, by analyzing factors affecting the safety of the interchange, presented a mathematical evaluation interchange security model based on the fuzzy credibility [13]. X. Jiang, according to North Third Ring Road highway interchange design in Guangzhou City, proposed the problems interchange security design [14]. $\mathrm{K}$. Zhang, analyzed the main factors affecting the safety impact of the interchange area, established the safety evaluation system of highway interchange area based on road conditions [15].

\subsection{Traffic conflict technique application}

In addition, traffic conflict analysis was as a method for non-traffic safety accidents, increasingly used on the road and intersections.

\subsubsection{Intersection}

U. Shahidah, replaced the crashes by simulating the conflicts for different road and traffic conditions, researched on the correlation between the number of traffic conflict and crash modification factors (CMFs), integrated empirical bayesian before-and-after methods, changed left turn signal priority at Toronto signalized intersections from permissive to protected-permissive [16]. A. Stevanovic, in order to optimize design in signalized intersection balancing safety and efficiency based on VISSIM multi-objective genetic algorithm to, used traffic conflict instead of traffic accident data, analysis the 12-intersection corridor on Glades Road in Boca Raton and two smaller synthetic network served, then optimized the signal timing[17]. L. Yuan, based on correlation analysis of road traffic conditions and traffic conflicts by gray relational theory in signalized intersections, established a relationship model between road traffic conditions and traffic conflicts rates [18].

\subsubsection{Basic segment}

J. Zhou, chosen the ratio between the number of traffic conflicts and the traffic volume in certain road section length as the index, simulating traffic conflicts by software VISSIM, selected fuzzy evaluation method to evaluate the freeway security situation, the examples verified effect [19]. Q. Shen, constructed the correlation relationship model between the traffic conflicts and interchange spacing, divided the level of risk by gray clustering method, and ultimately determined the new minimum interchange spacing [20]. S. Luo, focused on the criterion and grading standards, improved the traffic conflict theory, and provide a new means for the road traffic safety analysis and evaluation [21].

Traffic Conflict Technique was applied in intersection and road. In this paper, the traffic conflict would be used in researching on the interchange traffic safety, as a supplement of the interchange traffic safety research methods.

\section{Interchange traffic conflict type analysis}

Due to changes of structure or operations of road users in the interchange area, the road users would be close to each other at a certain time and space, leading to the rear vehicle must take evasive measures to change the driving state excluding the danger of collision. Interchange is the traffic facility keeping continuous flow; with the same direction of traffic, impact angle is less than $90^{\circ}$. According to the conflict angle partitioning method in SSAM analysis software, the interchange conflict was divided into two types: sideswipe conflict, rear-end the conflict. Sideswipe conflict belongs to $\left[15^{0}, 85^{0}\right]$, rear-end conflicts $\left[0^{0}, 15^{0}\right)$.

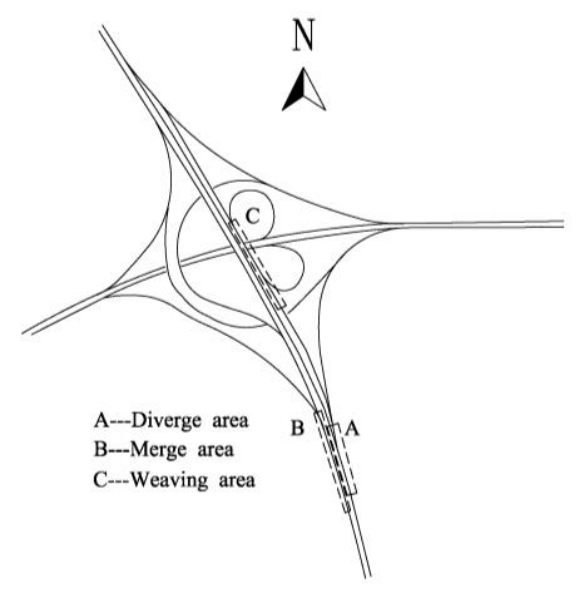

Figure 1. Maqun interchange investigation site

\section{Data collection and analysis}

\subsection{Data collection}

The investigation site was selected at the northeast of Nanjing Maqun interchange. It is the semi-clovers interchange type interchange, with a typical diverge, merge and weaving area. Unmanned aircraft was allowed 
to shoot video in the surroundings, including the wind force below grade 4, sunny weather, good light, no electromagnetic interference. Finally four hours high definition video traffic was obtained. Fig. 1 shown the case of observation points.

\subsection{Traffic conflict calculation method}

\subsubsection{The rear-end conflict}

Set the Tracker player steps as twenty frames per one second. According to the specifications, freeway lanedividing line, solid line six meters, dotted line nine meters, determined the video scale. Set the junction of interchange as the origin pixel of the coordinate system. The new mass A was selected the front vehicle of the existence conflict group, set the template tracking, set the mass A center at the rearmost end, started tracking, until traffic conflict disappears. A particle point coordinates of travel locus was $\left(\mathrm{X}_{\mathrm{A}}, \mathrm{Y}_{\mathrm{A}}\right)$. The trajectory point set was $\mathrm{L}_{\mathrm{A}}=\left\{\left(\mathrm{x}_{\mathrm{A} 1}\right.\right.$, $\left.\left.\mathrm{y}_{\mathrm{A} 1}\right),\left(\mathrm{x}_{\mathrm{A} 2}, \mathrm{y}_{\mathrm{A} 2}\right),\left(\mathrm{x}_{\mathrm{A} 3}, \mathrm{y}_{\mathrm{A} 3}\right), \ldots,\left(\mathrm{x}_{\mathrm{An}}, \mathrm{y}_{\mathrm{An}}\right)\right\}$.

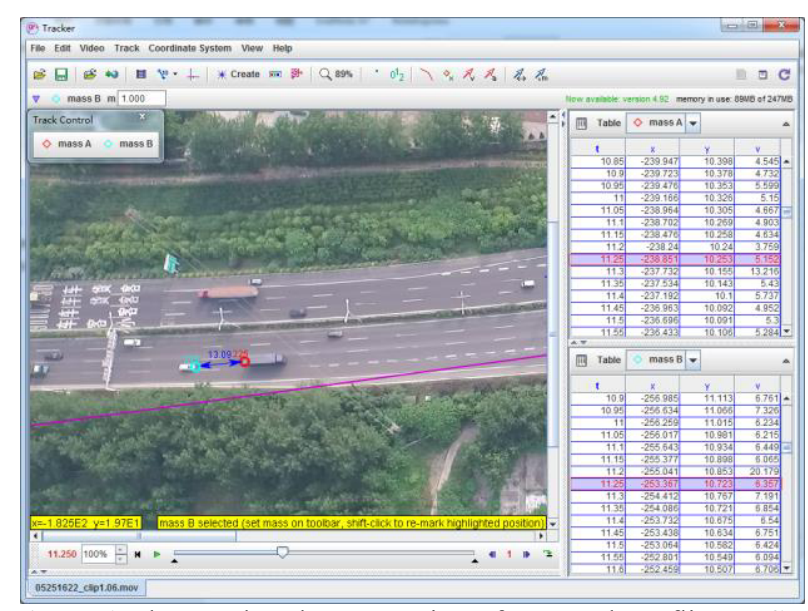

Figure 2.The Tracker demonstration of rear-end conflict TTC calculation.

Rewinding the Tracker video to the time of the conflict occurred, A was set as the origin of coordinates now. Meanwhile, the new mass B was selected the rear vehicle of the existence conflict group, and the mass B centre was set at the vehicle forefront. The track method was same to A. $B$ mass point coordinates of travel locus was $\left(X_{B}, Y_{B}\right)$. The trajectory point set was $\mathrm{L}_{\mathrm{B}}=\left\{\left(\mathrm{x}_{\mathrm{B} 1}, \mathrm{y}_{\mathrm{B} 1}\right),\left(\mathrm{x}_{\mathrm{B} 2}, \mathrm{y}_{\mathrm{B} 2}\right)\right.$, $\left.\left(\mathrm{x}_{\mathrm{B} 3}, \mathrm{y}_{\mathrm{B} 3}\right), \ldots,\left(\mathrm{x}_{\mathrm{Bn}}, \mathrm{y}_{\mathrm{Bn}}\right)\right\}$.

$\Delta \mathrm{L}_{\mathrm{B}}=$ the changes of distance of $\mathrm{B}$.

$$
\Delta L_{B}=\sqrt{\left(x_{B 2}-x_{B 1}\right)^{2}+\left(y_{B 2}-y_{B 1}\right)^{2}}
$$

$\Delta \mathrm{v}=$ one frame time traveling the changes distance.

The Tracker created the plot of relative distance changes and time.

$\mathrm{T}_{\mathrm{m}}=$ the horizontal axis value when the maximum slope of relative distance slow down.

$\mathrm{T}_{\mathrm{e}}=$ the maximum slope extended the horizontal axis point value.

$$
\Delta T=\left|T_{e}-T_{m}\right|
$$

Recorded the $\Delta \mathrm{T}$ as TTC data. The rear-end conflict TTC calculation method was shown in Fig. 2 and Fig. 3 by Tracker. In Fig. 3, the vertical axis " $r$ " was the distance of particle B away from the origin (particle A) each fame.

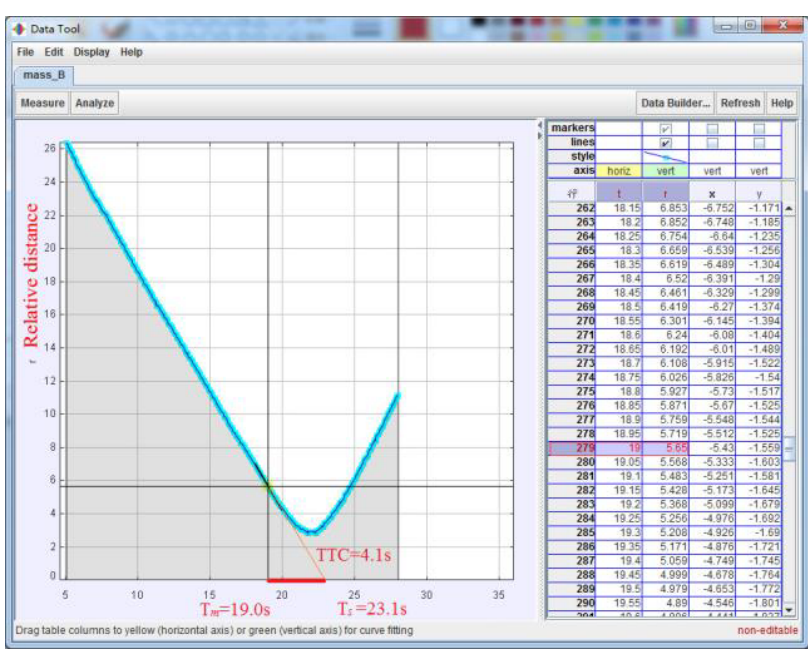

Figure 3. The rear-end conflict TTC calculation demonstration

\subsubsection{The sideswipe conflict}

For sideswipe conflict, since the two vehicles were in different lanes, it wasn't proved the conflict existence that the relative distance between two vehicles declined rapidly. Because even keeping this motion and doing nothing of evasive action, the intersection of the two vehicles might pass their track intersection independently. In rear-end collision, two vehicles had the same track. When the front vehicle speed declined, in the same time, the rear did not anything to change its speed, the crash would happen. But the sideswipe conflict tracks crossed a point (body length and width should be considered strictly speaking, a "block "). If one missed the only crossing point, no collision. So the sideswipe conflict calculation method was not same with rear-end.

Initial setups of the origin pixel of the coordinate system, the scale and the template were same with rearend conflict. But, the origin pixel of the coordinate system was fixed. The potential conflict point was the trajectory of the intersection of particle A and B. Output the plot of the speed and time both mass $\mathrm{A}$ and $\mathrm{B}$, and recode the speed suddenly slow down about $\mathrm{VA}$ and $\mathrm{V}_{\mathrm{B}}$, and then measured the distance about $\mathrm{L}_{\mathrm{A}}, \mathrm{L}_{\mathrm{B}}, \mathrm{S}_{\mathrm{A}}$ and $\mathrm{S}_{\mathrm{B}}$, respectively calculation $\mathrm{TTC}_{\mathrm{A}}, \mathrm{TTC}_{\mathrm{B}}$.

$\mathrm{V}_{\mathrm{A}}=$ the speed when mass $\mathrm{A}$ 's speed slow down suddenly.

$\mathrm{V}_{\mathrm{B}}=$ the speed when mass B's speed slow down suddenly.

$\mathrm{L}_{\mathrm{A}}=$ the length of vehicle $\mathrm{A}$.

$\mathrm{L}_{\mathrm{B}}=$ the length of vehicle $\mathrm{B}$.

$\mathrm{S}_{\mathrm{A}}=$ the distance between mass A's speed slow down suddenly and the potential conflict point.

$\mathrm{S}_{\mathrm{B}}=$ the distance between mass B's speed slow down suddenly and the potential conflict point.

$$
\begin{aligned}
& T T C_{A}=S_{A} / V_{A} \\
& T T C_{B}=S_{B} / V_{B}
\end{aligned}
$$


The results appeared in both cases.

a) $T T C_{A} \geqslant T T C_{B}$

If $\mathrm{TTC}_{\mathrm{A}} \leqslant \mathrm{TTC}_{\mathrm{B}}+\mathrm{L}_{\mathrm{B}} / \mathrm{V}_{\mathrm{B}}$, the conflict was effective, $\mathrm{TTC}=\mathrm{TTC}_{\mathrm{A}}$. Otherwise, the conflict was not valid.

b) $T T C_{A}<T T C_{B}$

If $\mathrm{TTC}_{\mathrm{B}} \leqslant \mathrm{TTC}_{\mathrm{A}}+\mathrm{L}_{\mathrm{A}} / \mathrm{V}_{\mathrm{A}}$, the conflict was effective, $\mathrm{TTC}=\mathrm{TTC}_{\mathrm{B}}$. Otherwise, the conflict was not valid.

And the sideswipe conflict TTC calculation method was shown in Fig. 4.

$$
\begin{array}{ll}
\text { a) } \mathrm{TTC}_{A} \geq \mathrm{TTC}_{B} & \text { b) } \mathrm{TTC}_{A}<\mathrm{TTC}_{B} \\
\text { And, } \mathrm{TTC}_{\mathrm{A}} \leqslant \mathrm{TTC}_{\mathrm{B}}+\mathrm{L}_{\mathrm{B}} / \mathrm{V}_{\mathrm{B}} & \text { And, } \mathrm{TTC}_{\mathrm{B}} \leqslant \mathrm{TTC}_{\mathrm{A}}+\mathrm{L}_{\mathrm{A}} / \mathrm{V}_{\mathrm{A}}
\end{array}
$$
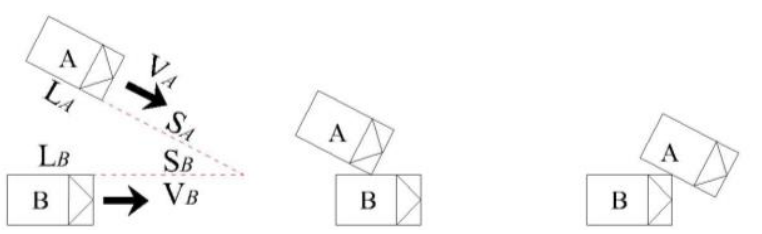

Figure 4. The sideswipe conflict TTC calculation method

\subsection{Error Analysis}

\subsubsection{Unmanned aircraft altitude error}

Unmanned aircraft, type INSPIRE 1, created by DJIInnovations in China, can hover on altitude of four hundred meters. In order to the quality of video, it worked on two hundred miters. Removal of the unstable part at the beginning and end of the video, ensure constant shooting height.

\subsubsection{Road surface gradient}

A small slope is usually designed on interchange, represented by $\alpha$, the design requirements $\alpha \leq 3^{0}$, according to extreme slope $\cos 3^{0}$ calculated, reduced projected length of the actual $0.14 \%$. It can be neglected on the same slope gradient.

\subsubsection{Tracer trajectory analysis}

Software working speed setting was twenty frames per one second. Then the rest parameters were set, including the reference line, selecting the junction as the original pixel position, angle, proportion, the scale. The tracking model template should contain the whole vehicle selecting trajectory in every frame. In the same position of interchange, the vehicle mark travel time was calculated by artificial check. Compared with the software displays, an error was $1.26 \%$, indicating that the result was statistically significant from software calculation.

\section{Traffic conflict severity subjective judgment}

Most observers have been trained in Nanjing at the School of Transportation, Southeast University. On request, the training crew also went to other places in or outside Nanjing. Training took one week, and about twelve people can be trained simultaneously as a group. The crew consists of three men whose task was teaching theory, background and practical skills to the video observers. These practical parts include appraisal of distance and speed, instruction from video tapes.

According highway accident statistics, with good road conditions and high speed, either a serious car crash accident or general scratch rear-end accidents could happened. So the traffic conflict subjective observer partitioned the severity of serious conflict and general conflict as the accidents varies in severity. Therefore, the interchange conflict was divided serious and general conflict by twelve observers. The next step, the samples of TTC should be test, and then the threshold would be defined

\section{Division the severity of traffic conflict}

\subsection{Sample Test}

Equation (5) was the minimum sample size required, according to the theory of error.

$$
N=\left(\frac{S K}{E}\right)^{2}
$$

Where: $\mathrm{N}$ is the smallest sample size;

$\mathrm{E}$ is the observed value of tolerance;

$\mathrm{S}$ is the estimated sample standard deviation;

$\mathrm{K}$ is determined by the confidence level of the coefficient.

\subsubsection{Sample Calculation}

After calculation, the sample standard deviations of various conflicts TTC are close to 1.0 , so " $\mathrm{S}$ " is 1.0 . Fixing the "S", with Equation (5), "E" was set interval of 0.2 and 0.1 second, the minimum sample size error situations was calculated for different confidence levels allowance.

\subsubsection{Traffic conflict severity classification}

According to the subjective judgment of learning, the severity of the conflict was divided into the serious conflict and general conflict. Observation of the classified data found that, serious conflict TTC value was in 2.5 second or less, the general conflict TTC value was between 2.5 second and 5 second.

The conflict sample distribution of different types and severity was shown in Table 1 . In the allowable error $\mathrm{E}=0.2,90 \%$ confidence level, the required minimum sample size was 68 . The actual amount sample of the various conflicts met the minimum sample size requirements.

Table 1. The actual conflict sample

\begin{tabular}{|c|c|c|}
\hline \multirow{2}{*}{ Severity } & \multicolumn{2}{|c|}{ Conflict Type } \\
\cline { 2 - 3 } & Sideswipe & Rear-end \\
\hline serious conflict & 106 & 71 \\
\hline general conflict & 153 & 84 \\
\hline
\end{tabular}




\subsection{TTC threshold calculation}

According to cumulative frequency distribution of TTC, the $85 \%$ position value was defined the upper bound of TTC threshold, then getting all TTC thresholds. For example, the following was calculation of serious rear-end conflict threshold.

The TTC data of serious conflict between 0.6 second and 3.4 second were divided fourteen groups, setting interval 0.2 second, and calculated the frequency and cumulative frequency of each data. Fig. 5 shown the TTC cumulative frequency distribution histogram. The $85 \%$ position value was between 2.8 second and 3.0 second, thus 2.8 second was defined as the serious conflict threshold.

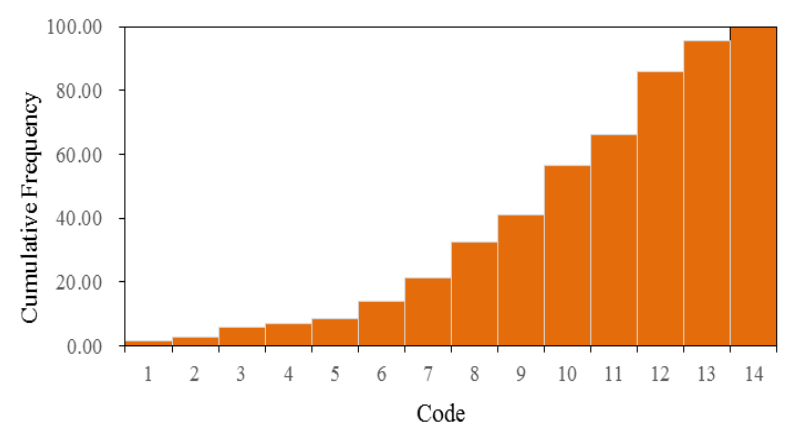

Figure 5. Serious rear-end conflict TTC cumulative frequency distribution histogram.

Using the same method, the other interchange conflict thresholds definition were shown in Table 2.

Table 2. The TTC threshold of interchange

\begin{tabular}{|c|c|c|c|c|}
\hline \multirow{2}{*}{$\begin{array}{c}\text { Conflict } \\
\text { Type }\end{array}$} & \multicolumn{2}{|c|}{ Rear-end } & \multicolumn{2}{c|}{ Sideswipe } \\
\cline { 2 - 5 } & $\begin{array}{c}\text { serious } \\
\text { conflict }\end{array}$ & $\begin{array}{c}\text { general } \\
\text { conflict }\end{array}$ & $\begin{array}{l}\text { serious } \\
\text { conflict }\end{array}$ & $\begin{array}{c}\text { general } \\
\text { conflict }\end{array}$ \\
\hline $\begin{array}{c}\text { threshold } \\
(\mathrm{TTC} / \mathrm{s})\end{array}$ & $0 \sim 2.8$ & $2.8 \sim 4.7$ & $0 \sim 2.3$ & $2.3 \sim 4.2$ \\
\hline
\end{tabular}

\section{Conclusion}

Based on the characteristics of freeway interchange continuous flow and collision angle less than right angle, the interchange conflicts were divided into sideswipe conflict and rear conflict. Twelve conflict observers were unified training with factors from traffic accident analysis, until cognitive consistency was reached. Then jointly determination was made on the type and severity of the conflict. With the help of unmanned aircraft and trajectory tracking software, the traffic conflict indicators TTC data were calculated. In the Plot of relative distance and time, one time was horizontal axis value when the maximum slope of relative distance changes slow down, and the other time was the maximum slope extended the horizontal axis point value; the absolute distance of two time was TTC value. Sideswipe conflicts, inappropriate using relative time, using minimum time of driving to the trajectory intersection point when the conflict occurred. The large error TTC data were removed. Based on statistics, the $85 \%$ position data of cumulative frequency was defined as the severity threshold. In sideswipe conflict, the serious and general threshold value was 2.3 second and 4.2 second; the rear-end conflict, threshold value was 2.8 second and 4.7 second. This paper have described the type of traffic conflict on interchange which is divided by traffic collision angle. In the future, further refined conflict severity may be defined by the specific environment.

\section{References}

1. C. Hydén, BLIT, 70, (1987)

2. S. Zhang, China traffic conflict technique (SCUP, Chengdu, 1998)

3. X. Liu, and H. Duan, HT. J. 14, 29 (1997)

4. W. Cheng, and J. Li, TSEIT. J. 4, 48 (2004)

5. W. Ceng, and G. Wang, KUST. J. ST. 30, 106 (2005)

6. Q. Xiang, J. Lu, Q. Wu, J. Lu, and Y. Ma, HTRD. J. 26, 132 (2009)

7. Q. Xiang, J. Lu, C. Lu, and X. Ge, Analysis and application of road traffic conflict technique, (Science Press, Beijing, 2008)

8. F. Pan, L. Zhang, and J. Lu, SJTU. J. 47, 259 (2013)

9. W. Guo, Z. Qu, and D. Wang, JLU. J. ET. 41, 35 (2011)

10. T. Guo, W. Deng, and J. Lu, TSEIT. J. 10, 76 (2010)

11. Y. Zhang, NT. J. 4, 3 (2010)

12. W. Wu, D. BJUT, (2009)

13. S. Fan, S. Zhao, Y. Yan, and H. Gao, HPC. J. 12, 42 (2009)

14. X. Jiang, and H. Yuan, Hig. J. 4, 167 (2006)

15. K. Zhang, D. CSUST. (2011)

16. U. Shahdah, F. Saccomanno, and B. Persaud, AAP. J. 71, 228 (2014)

17. Stevanovic, J. Stevanovic, and C. Kergaye, TRP C. J. 32, 159 (2013).

18. L. Yuan, H. Yuan, Q. Xiang, and J. Lu, TIS. J. 28, 117 (2010)

19. J. Zhou, Y. Chang, M. Guo, and G. Wang, CQJTU. J. NS. 30, 974 (2011)

20. Q. Shen, S. Yang, Y. Zhao, and H. Cao, SETP. 35, 160 (2015)

21. W. Zhou, and S. Luo, CJHT. J. 13, 82 (2000) 